\title{
Novel Concepts in Epileptogenesis and its Prevention
}

\author{
Lara E. Jehi • Annamaria Vezzani
}

Published online: 21 March 2014

(C) The American Society for Experimental NeuroTherapeutics, Inc. 2014

Around 50 million people in the world now have epilepsy, and it is estimated that 40-70 new cases per 100,000 individuals in the general population will acquire the disease every year in developed countries, with the risk being twice as high in developing countries [1]. An "epilepsy" diagnosis comes with a host of "comorbidities" ranging from significant developmental and cognitive complications, mood and anxiety disorders, and social stigma, to seizure-related injuries. This leads to the staggering annual health care cost of $\$ 15.5$ billion in the USA alone [2], and over $€ 13.8$ billion in Europe [3]. Mortality risk triples for all patients with epilepsy, and the rate of sudden death is increased at least 20 times in those with uncontrolled seizures [4]. The burden of the disease is huge, but there remains an unmet need to better treat it and, more importantly, prevent it. Despite booming rates in new drug development, and new surgical and diagnostic techniques, seizures remain uncontrolled in up to $40 \%$ of patients treated with medications, and in a similar proportion of patients who undergo resective epilepsy surgery [1]. Given this overwhelming disease burden and the limited success of available treatments, the efforts to reduce the development of epilepsy from the outset — preventing "epileptogenesis"—-become imperative.

Extensive work has been done over the last few decades to mature the concepts of epileptogenesis to where we stand today. The definition of the term "epileptogenesis" itself is constantly evolving, and the venues of research investigating

L. E. Jehi $(\bowtie)$

Cleveland Clinic Epilepsy Center, S51 9500 Euclid Avenue, Cleveland, OH 44195, USA

e-mail: jehil@ccf.org

A. Vezzani

Department of Neuroscience, IRCCS-Istituto di Ricerche Farmacologiche "Mario Negri”, Via G. La Masa 19, 20156 Milan, Italy it range from targeted, hypothesis-driven, animal model- or cell-based experimentation, to hypothesis-generating highthroughput "omics" approaches, including metabolomics and connectomics. This issue of Neurotherapeutics will review the most current understanding and research in this field, and provide a window to the future. Throughout the issue, prominent clinical researchers paired with established basic scientists provide a balanced and comprehensive discussion of each topic, illustrating both the mechanistic understanding and the clinical translation.

The issue will start with a historical perspective and a discussion of the evolution in our concepts and approaches to understanding epileptogenesis and its biomarkers. The first article, by Pitkänen and Engel [5] is designed to set the stage for the rest of the issue by laying out the main current accepted general principles and the challenges lying ahead. Next, a series of papers dissect the mechanisms of epileptogenesis in each of the major epilepsy etiologies. This section on "mechanisms" is designed to take the "epileptogenesis" discussion to a deeper and more specific level, focusing it on the most frequent and/or challenging epilepsy syndromes, including hippocampal sclerosis, tackled by Patterson et al. [6]; the development of epilepsy after head trauma, addressed by Pitkänen and Immonem [7]; and the defiant issue of epileptogenesis in "non-lesional" epilepsies, undertaken by Guerrini and Marini [8]. A separate article by Aronica and Crino [9] brings to the forefront the state-of-the-art understanding of epileptogenesis in developmental brain tumors and malformations of cortical development. Special pediatric considerations related to pediatric epilepsy syndromes are highlighted in a dedicated paper by Pardo et al. [10] This section is concluded by reviewing mechanisms of epileptogenesis in autoimmune epilepsies, as reviewed by Bien and Bauer [11].

The next major section in this issue focuses on an evaluation of the most promising potential epileptogenesis 
biomarkers currently available or under investigation. The wide range of molecular, genetic, electrophysiological, and neuroimaging biomarkers are each reviewed in detail. Each paper reviews the evidence and processes underlying specific proposed biomarkers, and discusses their clinical implications. More than anything, this section highlights the wide gaps between the current correlations of these "proposed biomarkers" with specific epilepsy syndromes/seizures and the still elusive goal of predicting future epileptogenesis for most epilepsies. The wealth of research linking epilepsy "comorbidities", such as depression and emotional challenges, to the processes of epileptogenesis will be reviewed at the end of this section by Kanner et al. [12].

The final section of this issue is devoted to therapeutic approaches, thus reflecting the culmination of the work that has been so far devoted to understanding how epileptogenesis develops (mechanisms) and how to assess it (biomarkers) into how this knowledge can ultimately improve patient care and outcomes. This section includes a set of articles dedicated to the experimental models used to search for effective antiepileptogenic agents by White and Löscher [13], followed by an overview of the most promising such drugs for clinical translation in humans by Kaminski et al. [14]. The particular issues and challenges of designing clinical trials to test potential antiepileptogenic agents are aptly discussed in a dedicated paper by Schmidt et al. [15].

We hope that this issue will provide a comprehensive overview of where the field of epilepsy currently stands in relation to understanding the process of epileptogenesis. More importantly, we hope that the body of work presented here begins to ask the right questions necessary to advance the field.

Required Author Forms Disclosure forms provided by the authors are available with the online version of this article.

\section{References}

1. World Health Organization. Epilepsy. Fact sheet number 999. October 2012. Available at: http://www.who.int/mediacentre/ factsheets/fs999/en/index.html. Accessed May 13, 2013.

2. National Center for Chronic Disease Prevention and Health Promotion. Targeting epilepsy improving the lives of people with one of the nation's most common neurological conditions at a glance 2011. June 6, 2011. Available at: http://www.cdc.gov/chronicdisease/ resources/publications/AAG/epilepsy.htm. Accessed May 13, 2013.

3. Gustavsson A, Svensson M, Jacobi F, Allgulander C, Alonso J, Beghi E, et al. Cost of disorders of the brain in Europe 2010. Eur Neuropsychopharmacol 2011;21:718-779.

4. Institute of Medicine, Board on Health Sciences Policy. Epilepsy Across the spectrum: Promoting health and understanding. March 30, 2012. Available at: http://www.iom.edu/Reports/2012/EpilepsyAcross-the-Spectrum.aspx. Accessed June 1, 2012.

5. Pitkänen A, Engel J, Jr. Past and present definitions of epileptogenesis and its biomarkers. Neurotherapeutics 2014.

6. Patterson KP, Baram TZ, Shinnar S. Origins of temporal lobe epilepsy: Febrile seizures and febrile status epilepticus. Neurotherapeutics 2014.

7. Pitkänen A, Immonem R. Epilepsy related to traumatic brain injury. Neurotherapeutics 2014.

8. Guerrini R, Marini C. Genetic epilepsy syndromes without structural brain abnormalities: Clinical features and experimental modes. Neurotherapeutics 2014.

9. Aronica E, Crino PB. Epilepsy related to developmental tumors and malformations of cortical development. Neurotherapeutics 2014.

10. Pardo CA, Nabbout R, Galanopoulou AS. Mechanisms of epileptogenesis in pediatric epileptic syndromes: Rasmussen encephalitis, infantile spasms, and febrile-infection-related epilepsy syndrome (FIRES). Neurotherapeutics 2014.

11. Bien CG, Bauer J. Autoimmune epilepsies. Neurotherapeutics 2014.

12. Kanner, AM, Mazarati A, Koepp M. Biomarkers of epileptogenesis: Psychiatric comorbidities? Neurotherapeutics 2014.

13. White HS, Löscher W. Searching for the ideal antiepileptogenic agent in experimental models: Single treatment versus combinatorial treatment strategies. Neurotherapeutics 2014.

14. Kaminski RM, Rogawski MA, Klitgaard H. The potential of antiseizure drugs and agents that act on novel molecular targets as antiepileptogenic treatments. Neurotherapeutics 2014.

15. Schmidt D, Friedman D, Dichter MA. Anti-epileptogenic clinical trial designs in epilepsy: Issues and options. Neurotherapeutics 2014. 